\title{
SOME HARDY INEQUALITIES ON HALF SPACES FOR GRUSHIN TYPE OPERATORS
}

\author{
YING-XIONG XIAO
}

Abstract. We prove some sharp Hardy type inequalities on half spaces for Grushin type operators like $\Delta_{x}+(1+\gamma)^{2}|x|^{2 \gamma} \Delta_{y}$ with $\gamma>0$.

Mathematics subject classification (2010): Primary 26D10, 35H20.

Keywords and phrases: Hardy inequality, Grushin type operators.

\section{REFERENCES}

[1] L. D'Ambrosio, Hardy inequalities related to Grushin type operators, Proc. Amer. Math. Soc. 132 (2004), 725-734.

[2] L. D'Ambrosio and S. Lucente, Nonlinear Liouville Theorems for Grushin and Tricomi Operators, J. Differential Equations 193 (2003), 511-541.

[3] J. Dou, Q. Guo, P. Niu, Hardy inequalities with remainder terms for the generalized BaouendiGrushin vector fields, Math. Ineq. Appl. 13, 3 (2010), 555-570.

[4] B. Franchi, C. E. Gutiérrez, R. L. Wheeden, Weighted Sobolev-Poincaré inequalities for Grushin type operators, Comm. Partial Differential Equations 19 (1994), 523-604.

[5] S. Filippas, A. Tertikas, J. Tidblom, On the structure of Hardy-Sobolev-Maz'ya inequalities, J. Eur. Math. Soc. 11, 6 (2009), 1165-1185.

[6] G. B. Folland, E. M. Stein, Hardy spaces on homogeneous groups, Princeton University Press, Princeton, NJ, 1982.

[7] V. MaZ'Ya, T. Shaposhnikova, A Collection of Sharp Dilation Invariant Integral Inequalities for Differentiable Functions, in "Sobolev Spaces in Mathematics I: Sobolev Type Inequalities", ed. V. Maz'ya, International Mathematical Series 8, Springer, 2009, 223-247. 